\title{
A Survey on Detecting License Plates from Low Quality Images
}

\author{
S. Neeraja \\ Master of Computer Science and Engineering \\ Government College of Technology Coimbatore, \\ India
}

\begin{abstract}
In real-time, identifying license plates from low quality images affected by multiple factors, such as severe brightness condition, tough background, distinct weather conditions, darkness, and appearance distortions is a difficult process. This paper gives a comparative analysis of different license plate detection (LPD) techniques in terms of their detection ratios. Super-resolution and enhancement techniques are discussed to overcome the problems faced by license plate recognition systems such as detecting license plates from blurred images. It is realized that image enhancement techniques are superior to image super-resolution techniques for improving low quality images.
\end{abstract}

\section{Keywords}

LPD, low resolution, super-resolution, image enhancement

\section{INTRODUCTION}

Intelligent transportation systems play an essential role in supporting smart cities. One of the keystone components of intelligent transportation systems is vehicle license plate recognition, which empowers identification of each vehicle. License plate recognition system mainly consists of four steps such as vehicle image capture, license plate detection (LPD), character segmentation and character recognition Figure 1. LPD is the basic component of vehicle license plate recognition as its performance, largely decide the overall accuracy and processing speed of the recognition system as well as provides support for intelligent transportation systems in smart cities [1]. Two decades has been exhausted for improving LPR systems harmonious to situations but there are still several challenges in achieving high detection and recognition rates. One such factor is low quality inclined by the following reasons: (i) sarcastic outdoor brightness conditions during image acquirement such as effects from headlight and sunshine, (ii) Low quality license plate images which often hold damaged or stained license plates and nonlicense plate characters printed on vehicles, and (iii) Perspective distortions due to distance or standpoint variations [11].

\author{
S. Rathi, PhD \\ Assistant Professor (Senior Grade) \\ Department of Computer Science and Engineering \\ Government College of Technology, Coimbatore, \\ India
}

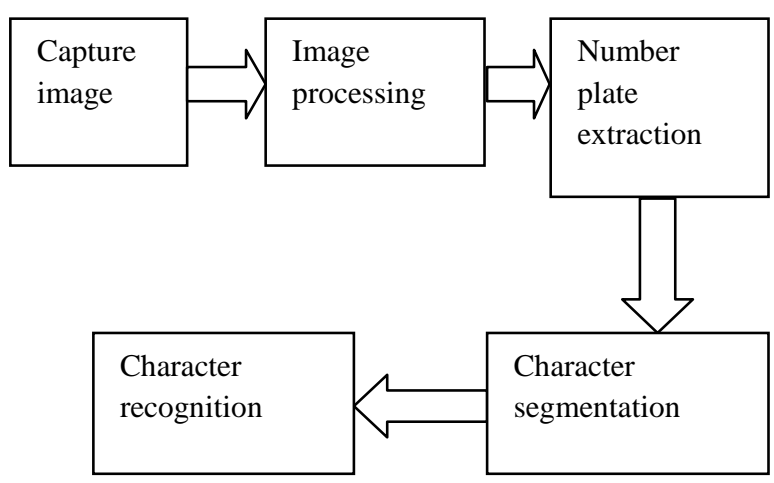

Figure 2. Basic block diagram for license plate recognition system

Many closed-circuit television (CCTV) surveillance cameras used in traffic monitoring systems, car parking system, electronic toll collection system, crime detection etc uses various resolutions hence it is a major problem for detecting license plates from low quality images. Spatial resolution enhancement of license plate images in real scenarios plays an essential role in the fields of criminal investigation and forensic science [10]. Among all the applicable techniques, super-resolution (SR) is one of the most effective image magnification techniques extensively used today because it has a lot of desired properties, like various motion models (global or local motion model), incorporating suitable a priori knowledge of the target image, and yielding much more results than many other methods [8]. Motion estimation is applied to reconstruct the high resolution (HR) image. However, multi-frame techniques are only beneficial for a diminutive increase in resolution. Edge based insertion methods are developed with the goal of improving artifacts around margin. These methods employ directional edge intelligence to sustain edge sharpness in the HR image [12].

\section{LITERATURE SURVEY}

LPD is the basic component of vehicle license plate recognition. An enumerate number of LPD methods have been proposed over the past two decades. Most of the preceding methods perform well only under certain predefined conditions. Some usual restrictions include fixed illumination, license plates with little blur or distortion from standpoint changes, relatively simple backgrounds and the presence of only a single license plate in an image. Various methods are proposed to find the exact location of the license plates from different resolutions of images. Some of them are listed such as i) Magnitude of the vertical gradients (angles) ii) Rectangular shift window iii) Region based method iv) Adaptive image segmentation technique v) Block based edge density prediction method vi) Vertical edge detection 
algorithm vii) LPD algorithm based on MSER concept viii) Morphological techniques ix) Neural networks x) Template matching techniques.

In the literature survey the different techniques used for various resolutions of images with super-resolution and enhancement techniques to improve low quality images are discussed:

\subsection{LPD Techniques for various resolutions of images}

A robust and effectual method for license plate detection with the intention of exactly localizing vehicle license plates from difficult scenes in real time is proposed by Yule Yuan et al. [1]. A simple yet effectual image downscaling process is first proposed to essentially accelerate license plate localization without sacrificing detection performance compared with that achieved using the primitive image. A modern line density filter approach is designed to obtain candidate regions, thereby reducing the area to be identified for license plate localization. Moreover, a cascaded license plate classifier based on linear support vector machines using color saliency characteristic is introduced to recognize the exact license plate among the candidate regions. The license plates with $1082 * 728$ image resolutions were detected with detection ratio of $96.62 \%$.

License plate detection is an essential stage in vahan license plate recognition for machine-driven transportation system proposed by D. Zheng et al. [2]. This paper presents a real time and robust method of license plate location. License plate region contains rich edge and texture information. Initially, extract the vertical margin of the automobile image using image enhancement and Sobel operator, then remove most of the rear and noise margin by an efficacious algorithm, and eventually search the plate region by a rectangle window in the remaining edge image. Finally, segment the plate out from the primitive automobile image. The license plates with $384 *$ 288 image resolutions were detected with detection ratio of $99.7 \%$.

This method consists of four major parts: image enhancement, the first round of voting to get the candidate blocks, boundary adjustment, and the second round of voting to get the real plate image proposed by Yong Zhao et al. [3]. First round of voting is done for the candidate blocks and the second round of voting is supported on several characteristic of the license plate. It uses the horizontal projection to decide the horizontal boundaries of the license plate and the vertical projection to decide the vertical boundaries of the plate. The license plates with $1624 * 1232$ resolutions were detected with detection ratio of $98 \%$.

License plates detection is extensively considered a solved problem, with many systems already in operation proposed by Wengang Zhou et al. [4]. However, the existing algorithms or systems are under some restraint conditions. There are still many challenges for license plate detection in open environment, such as various observation angles, background disorder, scale changes, multiple plates, uneven brightness, and so on. Here a modern scheme to automatically locate license plates by Principal Visual Word (PVW), discovery and local feature matching had been intend. Observing that characters in distinct license plates are duplicates of each other, the bag-of-words (BoW) model has been applied in partial-duplicate image search. Unlike the classic BoW model, for each plate character, the PVW characterized with geometrical context has been automatically detected. Given a new image, the license plates are extracted by matching local features with PVW. Besides license plate detection, it can also be extended to detection of logo's and trademarks. Due to invariance bravery of scale-invariant feature transform feature, these methods can adaptively deal with different changes in the license plates such as rotation, scaling, brightness, etc. PVW are diminutive parts of image that carry information related to characteristics (color, shape or texture) or changes in pixels. The license plates with $896 * 592$ resolutions were detected with detection ratio of $84.8 \%$.

A modern algorithm for license plate detection in complex scenes, particularly for the all-day traffic surveillance environment proposed by $\mathrm{Bo} \mathrm{Li}$ et al. [5]. Unlike low-level feature based methods, it is motivated by component-based models for object detection. The detection process is divided into three steps, namely, decomposition, modeling, and deduction. Initially, observing that one license plate is decompounded into several constituent characters, the maximally stable extremal region detector is used to extract candidate characters in images. Secondly, Conditional Random Field (CRF) models are constructed on the candidate characters in neighborhoods. This way, spatial and visual relationships among the characters is incorporated in CRF in the form of probability distribution. Finally, true bounding boxes of license plates are estimated through the belief propagation inference on CRF. Both visible as well as constructional features of license plates are fully exploited during detection. Hence, this approach can fit to different environmental factors, such as disorder background and brightness variation. The license plates with $720 * 576$ resolutions were detected with detection ratio of $97.1 \%$.

A license plate detection method supported on the gradient intelligence and a cascade discovery framework has been introduced by Runmin Wang et al. [6]. The proposed method consists of three main modules: image preprocessing, license plate detection and license plate verification. Considering the diversities of the license plates, first get the gradient images to unify plate forms through image preprocessing. The license plate will be detected roughly by using the cascade AdaBoost classifier in the next step. Finally, several heuristic discrimination strategies and a voting-based method are used to authenticate the candidate license plates. Voting is performed on different features of the license plates such as height and width ratios, number of characters on the license plates etc. The license plates with $1920 * 1088$ resolutions were detected with detection ratio of $87.29 \%$.

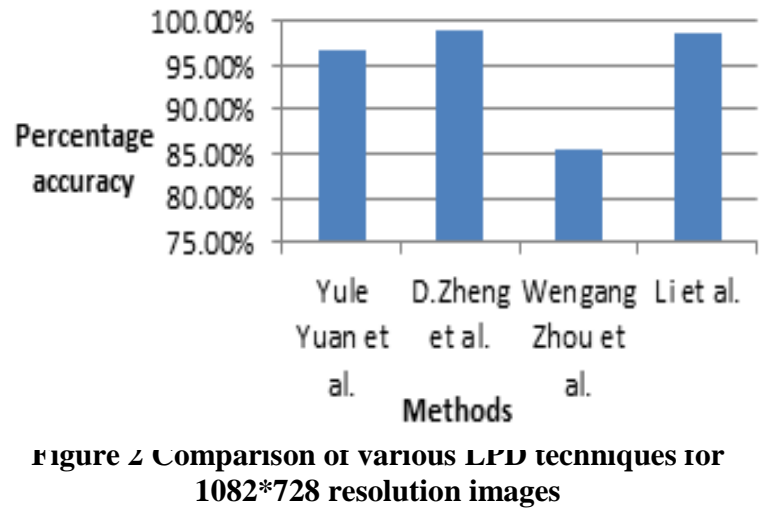




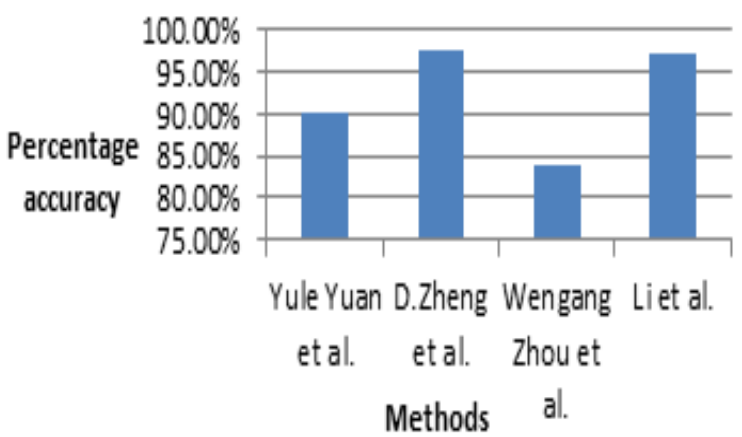

Figure 3 Comparison of various LPD techniques for 896*592 resolution images

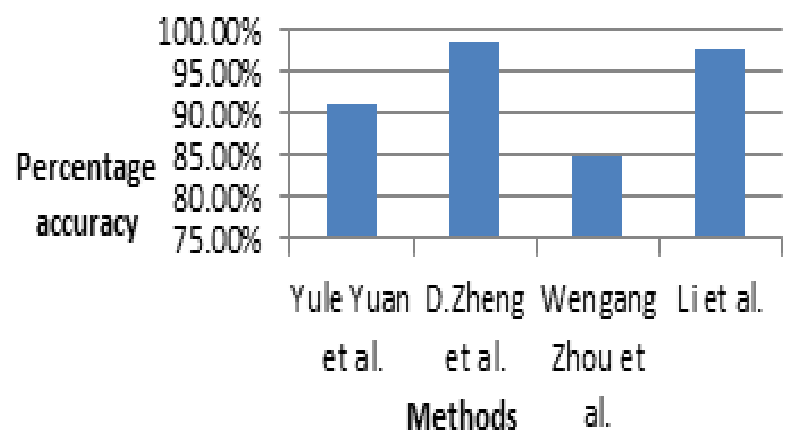

Figure 4 Comparison of various LPD techniques for $720 * 576$ resolution images

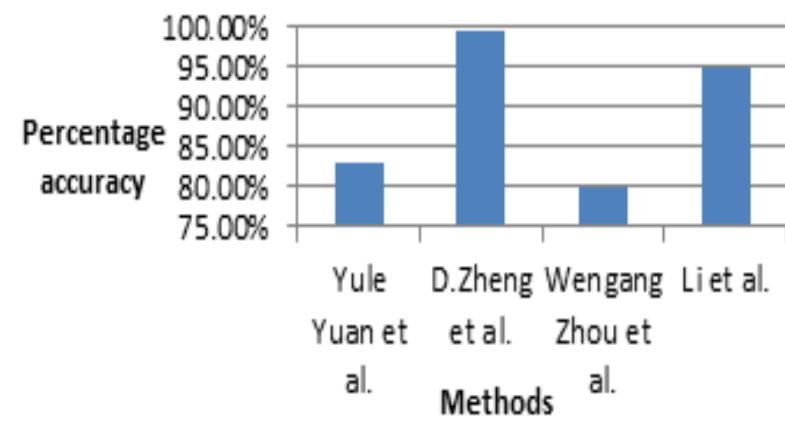

Figure 5 Comparison of various LPD techniques for $384 * 288$ resolution images

A comprehensive review of the state-of-the-art techniques for ALPR has been presented by Shan Du et al. [7]. Different ALPR techniques according to the characteristic and a comparison among them in terms of pros, cons, recognition accuracy, and processing speed had been categorized. Some of the techniques for license plate extraction are: Boundary or edge information, Global image information, Texture Features, Color Features, Character Features, combining two or more characteristic. Since each of them has their own drawback, the best among them has been chosen and presented. The license plate ordinarily has a rectangular shape with an understood aspect ratio; it can be extracted by finding all rectangles in the image. Boundary or Edge detection methods are usually used to find these rectangles. Edge-based methods are simple and fast. Sobel filter is used to detect edges. Sobel operator detects both horizontal and vertical edges. It is a separate differentiation operator for computing approximation of slop of image intensity function. It commonly uses $3 * 3$ or $1 * 3$ convolution masks. It also gives more precision when compared to other operators. Boundary or edge information produces detection ratio of $99.6 \%$.

Table 1. Summarization of the LPD techniques

\begin{tabular}{|c|c|c|c|c|}
\hline $\begin{array}{l}\text { EXISTING } \\
\text { SYSTEMS }\end{array}$ & TECHNIQUES & STRENGTH & WEAKNESS & ACCURACY \\
\hline $\begin{array}{l}\text { Yule Yuan et al. } \\
2017\end{array}$ & $\begin{array}{l}\text { Image downscaling, } \\
\text { LDF, SVM }\end{array}$ & $\begin{array}{l}\text { 1) Outperforms in terms } \\
\text { of detection accuracy and } \\
\text { runtime. }\end{array}$ & $\begin{array}{l}\text { 1) Efficiency reduces to } \\
91.27 \% \text { on } 896 * 592 \\
\text { resolutions of images. }\end{array}$ & $\begin{array}{l}\text { The license plates with } \\
1082 * 728 \text { image } \\
\text { resolutions were } \\
\text { detected with detection } \\
\text { ratio of } 96.62 \% \text {. }\end{array}$ \\
\hline $\begin{array}{l}\text { D. Zheng et al. } \\
2005\end{array}$ & $\begin{array}{l}\text { Image enhancement, } \\
\text { Vertical edge } \\
\text { extraction, Plate } \\
\text { search and } \\
\text { segmentation }\end{array}$ & $\begin{array}{l}\text { 1) High license plate } \\
\text { location rates. } \\
\text { 2) Supports images with } \\
\text { shadows of sunlight. }\end{array}$ & $\begin{array}{l}\text { 1) License plates should } \\
\text { be of same size. }\end{array}$ & $\begin{array}{l}\text { The license plates with } \\
384 * 288 \text { image } \\
\text { resolutions were } \\
\text { detected with detection } \\
\text { ratio of } 99.7 \% \text {. }\end{array}$ \\
\hline
\end{tabular}




\begin{tabular}{|c|c|c|c|c|}
\hline $\begin{array}{l}\text { YongZhao et al. } \\
2011\end{array}$ & Voting based & $\begin{array}{l}\text { 1) Fast for HD images. } \\
\text { 2) Detects plates from } \\
\text { shadows, sun reflections. }\end{array}$ & $\begin{array}{l}\text { 1) Processing time } \\
\text { increases from } 230 \mathrm{~ms} \\
\text { for less than } 70 * 20 \\
\text { resolution images. } \\
\text { 2) Accuracy reduces } \\
\text { from } 90 \% \text { for less than } \\
70 * 20 \text { resolution } \\
\text { images. }\end{array}$ & $\begin{array}{l}\text { The license plates with } \\
1624 * 1232 \text { resolutions } \\
\text { were detected with } \\
\text { detection ratio of } 98 \% \text {. }\end{array}$ \\
\hline $\begin{array}{l}\text { Wengang Zhou } \\
\text { et al. } 2012\end{array}$ & $\begin{array}{l}\text { Principal Visual } \\
\text { Word }\end{array}$ & $\begin{array}{l}\text { 1) Detects multiple } \\
\text { plates. } \\
\text { 2) Deals with various } \\
\text { angles, illuminations and } \\
\text { scaling. } \\
\text { 3) Plate recognition is } \\
\text { partially addressed. }\end{array}$ & $\begin{array}{l}\text { 1) Does not support } \\
\text { images less than } 15 \\
\text { pixels. } \\
\text { 2) Too severe when } \\
\text { distortion of angle is } \\
\text { changes from } 128 \\
\text { degree. }\end{array}$ & $\begin{array}{l}\text { The license plates with } \\
896 * 592 \text { resolutions } \\
\text { were detected with } \\
\text { detection ratio of } \\
84.8 \% \text {. }\end{array}$ \\
\hline $\begin{array}{l}\text { Bo Li et al. } \\
2013\end{array}$ & $\begin{array}{l}\text { Conditional random } \\
\text { field model }\end{array}$ & $\begin{array}{l}\text { 1) Supports all day } \\
\text { complex traffic scenarios. } \\
\text { 2) Character recognition } \\
\text { is partially addressed. }\end{array}$ & $\begin{array}{l}\text { 1) Fails on unclear and } \\
\text { obscure character } \\
\text { images. } \\
\text { 2) Fails on overexposed } \\
\text { images. }\end{array}$ & $\begin{array}{l}\text { The license plates with } \\
720 * 576 \text { resolutions } \\
\text { were detected with } \\
\text { detection ratio of } \\
97.1 \% \text {. }\end{array}$ \\
\hline $\begin{array}{l}\text { Runmin Wang } \\
\text { et al.2014 }\end{array}$ & AdaBoost classifier & $\begin{array}{l}\text { 1) Detects multiple } \\
\text { license plates from an } \\
\text { image. } \\
\text { 2) Uses less number of } \\
\text { cascade AdaBoost } \\
\text { classifiers. }\end{array}$ & $\begin{array}{l}\text { 1) Some candidate } \\
\text { regions of images may } \\
\text { not be scanned since } \\
\text { scale factor of } \\
\text { AdaBoost classifier is } \\
\text { selected artificially. } \\
\text { This may lead to license } \\
\text { plates undetected. }\end{array}$ & $\begin{array}{l}\text { The license plates with } \\
1920 * 1088 \text { resolutions } \\
\text { were detected with } \\
\text { detection ratio of } \\
87.29 \% \text {. }\end{array}$ \\
\hline $\begin{array}{l}\text { Shan Du et al. } \\
2013\end{array}$ & $\begin{array}{l}\text { Boundary or edge } \\
\text { information }\end{array}$ & $\begin{array}{l}\text { 1) Simplest, fast and } \\
\text { straightforward. }\end{array}$ & $\begin{array}{l}\text { 1) Too sensitive to } \\
\text { unwanted edges. }\end{array}$ & $99.6 \%$. \\
\hline
\end{tabular}

\subsection{Super resolution and enhancement techniques for improving the quality of images}

Traffic video surveillance cameras are extensively used today. However, resolution of the output video is very low and it is difficult to recognize the license number of the vehicles that broke traffic regulations. The Super-resolution image reconstruction techniques thus can be used to solve this problem. The goal of super resolution (SR) is to improve the spatial resolution of a low-resolution (LR) image by certain factor using either single or multiple LR input images. Singleimage SR methods can be further parted into two main categories: reconstruction-based methods and learning-based methods. Reconstruction-based methods consist in explaining a constraint for the HR image such that the reconstruction quality is increased. Learning-based methods, also known as hallucination methods, use a machine learning step to calculate the details of the super-resolved image. Image restoration techniques are distinct form image enhancement techniques. Image restoration is the function of taking a spoiled or noisy image and estimating a clean image. Image enhancement is enhancing the characteristics of the image that makes images more pleasing but not necessarily to exhibit a realistic data. The enhancement step is essential for license plate detection to improve the quality of images.

A novel anchor-based local learning method for single image super resolution SISR by joint learning of the feature space partition and local regressors is proposed by Kai Zhang et al. [8]. The joint learning problem is converted into a mixture of experts MoE model and an expectation-maximization (EM) algorithm is adopted. The MoE consists of two components: gating network learning and local regressors learning. An EM algorithm trains MoE on a large set of LR/HR patch pairs. The proposed method can use much less local models and time to achieve comparable or superior results to state-of-theart SISR methods, providing a highly practical solution to real applications.

A deep learning process for single image super-resolution (SR) has been proposed by Chao Dong et al. [9]. This process directly learns an end-to-end mapping between the low/highresolution images. The mapping is represented as a deep convolutional neural network $(\mathrm{CNN})$ that takes the low resolution image as the input and outputs the high resolution one. The ancient-fashioned sparse-coding-based SR methods can also be sighted as a deep convolutional network. But dissimilar old-fashioned method that handles each component separately, this process together optimizes all layers. The deep 
CNN has a light weight construction, yet exhibited state-ofthe-art restoration quality, and achieves fast speed for practical on-line usage. Different network structures and parameter settings are explored to realize trade-offs between performance and speed.

Spatial resolution enhancement of license plate images in actual scenarios plays an essential role in the fields of malefactor investigation and forensic science. A learningbased single-image super-resolution method that uses a priori wisdom of the input as the plate images captured at poor quality and very low resolution is presented by Alexandre Nata Vicente et al. [10]. The proposed method employs a decision tree to classify the input image and the classification results are used to weight the image patches in the reconstruction step. Additionally, histogram equalization is performed to improve the performance of the classifier. Experiments conducted on synthetic and real-world image exhibit that the proposed method is capable of producing sufficient results.

One of the major sources of poor results in License plate recognition is low quality of images affected by multiple factors, such as severe brightness condition, complicate background, distinct weather conditions, darkness, and perspective distortions. A modern mathematical model based on Riesz fractional operator for enhancing details of edge information in License plate images to improve the performances of text detection and recognition methods have been introduced by K. S Raghunandan et al. [11]. The proposed model executes convolution operation of Riesz fractional derivative over each input image by enhancing the edge robustness in it. Experimental results on enhancement show that the proposed model outperforms the existing baseline enhancement techniques in terms of quality metric. Further, experimental results on text detection and recognition show that text detection and recognition rates are amended significantly after enhancement compared to before enhancement. Fractional calculus is a greater area of studying nonlinearity in mathematics, which considers real and complex number powers of the differentiation operator to solve problems. It can be seen that the benefit of such fractional derivatives are explored in several applications, such as engineering, automatic control, finite impulse response filter designs, and biomedical applications. It is understood that image enhancement is a process of studying unexpected changes in an image to improve the perception of image quality. It is also real that derivatives are well understood functions for extracting such unexpected changes according to literature. Since fractional derivatives imply such derivations using real and complex number power, it is awaited to improve poor quality images affected by multiple factors compared to ordinary derivatives. This helps in solving several image processing and pattern recognition issues, one of which is enhancing the quality of license plate images since usually license plate images have multiple distortions. Riesz fractional order derivative is used for image sharpening by adjusting the fractional order of derivative; hence Riesz fractional order derivative for enhancing the quality of license plate images has been explored. The second order Riesz fractional differential operator is enough to realize the target.

The goal of super-resolution (SR) is to grow the spatial resolution of a low resolution (LR) image by a certain factor using either single or multiple LR input images. A machine learning-based approach to rebuild a high-resolution (HR) image from a single LR image has been presented by Paheding Sidike et al. [12]. Inspired by the human visual cortex system, which is caring to high-frequency (HF) components in an image, the model is endeavored based on this concept by training a neural network to estimate the missing HF components that contain structural details. In this process, distinct directional margin responses at each pixel are weigh to sustain more complete HF information and then a regularized extreme learning regression model is trained using a set of LR and HR images. Finally, the trained system is applied to a LR image to produce HR image.

Table 2. Summarization of super-resolution $\&$ enhancements techniques

\begin{tabular}{|l|l|l|l|l|}
\hline $\begin{array}{l}\text { EXISTING } \\
\text { SYSTEMS }\end{array}$ & TECHNIQUES & STRENGTH & WEAKNESS & PERFORMANCE \\
Kai Zhang et al. \\
2016 & $\begin{array}{l}\text { Linear regression, } \\
\text { joint learning }\end{array}$ & $\begin{array}{l}\text { 1) The proposed approach } \\
\text { provides a highly practical } \\
\text { solution to real SISR } \\
\text { applications. } \\
\text { 2) It produces more appealing } \\
\text { results than many state-of-the- } \\
\text { art algorithms both } \\
\text { quantitatively and } \\
\text { qualitatively. }\end{array}$ & $\begin{array}{l}\text { 1) Time complexity of the } \\
\text { proposed method mainly } \\
\text { depends on the number of } \\
\text { anchor points. }\end{array}$ & $\begin{array}{l}\text { PSNR - 29.66 dB } \\
\text { SSIM - 0.793 }\end{array}$ \\
\hline $\begin{array}{l}\text { Chao Dong et al. } \\
2016\end{array}$ & $\begin{array}{l}\text { Deep } \\
\text { Convolutional } \\
\text { Neural networks }\end{array}$ & $\begin{array}{l}\text { 1) Simple and robust. } \\
\text { 2) Different upscaling factors } \\
\text { canplied. }\end{array}$ & $\begin{array}{l}\text { 1) More number of } \\
\text { convolutional layers leads } \\
\text { to complexity. } \\
\text { 2) It requires more training } \\
\text { time. }\end{array}$ & $\begin{array}{l}\text { PSNR }-27.50 \mathrm{~dB} \\
\text { SSIM - 0.7513 }\end{array}$ \\
\hline
\end{tabular}




\begin{tabular}{|c|c|c|c|c|}
\hline $\begin{array}{l}\text { Alexandre Nata } \\
\text { Vicente et al. } \\
2016\end{array}$ & $\begin{array}{l}\text { Decision tree } \\
\text { classifier }\end{array}$ & $\begin{array}{l}\text { 1) Generates images with } \\
\text { better results in terms of edge } \\
\text { definition and contrast. } \\
\text { 2) Degraded images can also } \\
\text { provide results. }\end{array}$ & $\begin{array}{l}\text { 1) The use of some other } \\
\text { classifiers like SVMs, } \\
\text { AdaBoost is not evaluated. } \\
\text { 2) Dataset includes only } \\
300 \text { images. }\end{array}$ & $\begin{array}{l}\text { PSNR }-25.11 \mathrm{~dB} \\
\text { SSIM }-0.857\end{array}$ \\
\hline $\begin{array}{l}\text { K. S. } \\
\text { Raghunandan et } \\
\text { al } 2016\end{array}$ & $\begin{array}{l}\text { Riesz fractional } \\
\text { operator }\end{array}$ & $\begin{array}{l}\text { 1) Improves the performances } \\
\text { of license plate detection and } \\
\text { recognition. } \\
\text { 2) Includes images which are } \\
\text { blur, affected by fog, sunlight } \\
\text { and contains complex } \\
\text { backgrounds. }\end{array}$ & $\begin{array}{l}\text { 1) Recognition methods } \\
\text { report poor results } \\
\text { compared to detection } \\
\text { methods (i.e) recognition } \\
\text { rates are } 76.3 \% \text { while } \\
\text { detection ratio are } 81.9 \% \text {. }\end{array}$ & $\begin{array}{l}\text { PSNR - } 22.44 \mathrm{~dB} \\
\text { SSIM - } 0.996\end{array}$ \\
\hline $\begin{array}{l}\text { Paheding Sidike } \\
\text { et al } 2017\end{array}$ & $\begin{array}{l}\text { Regularized } \\
\text { Extreme Learning } \\
\text { Regression }\end{array}$ & $\begin{array}{l}\text { 1) Much less complicated. } \\
\text { 2) Can be easily implemented } \\
\text { in parallel processing } \\
\text { architectures. }\end{array}$ & $\begin{array}{l}\text { 1) Exploration of } \\
\text { directional edge features in } \\
\text { terms of high-order filter } \\
\text { types and noise sensitivity } \\
\text { reduces the performance. }\end{array}$ & $\begin{array}{l}\text { PSNR }-36.50 \mathrm{~dB} \\
\text { SSIM }-0.9750\end{array}$ \\
\hline
\end{tabular}

\section{DISCUSSION}

From the literature survey mentioned above, a comparative study and analysis on different existing techniques have been made and concluded that each of them has its own strength and weakness. The experimental analysis of each technique differs from one another and hence the best suited techniques can be applied for the enhancement of the low quality images. Although the hardware requirements for each technique vary from one another, the software's used may be the same. Table 1 shows the various advantages and disadvantages of different license plate detection methods and table 2 for superresolution and enhancement techniques. Figs.[2,3,4,5] shows the comparison of various LPD techniques used for different resolutions of images. From the Figs.[2,3,4,5], it can be found that the technique used by $D$. Zheng et al. [2] works effectively for $384 * 288$ resolution of images. A single image based learning method for very low quality license plate images which explores the prior knowledge that the images contain only digits with known format is one of the image super-resolution techniques used widely. In table 2, the standard quality measures, namely, Peak Signal to Noise Ratio (PSNR) and Mean Structural Similarity (MSSIM) are used to measure the quality of the image. PSNR is calculated by the mean squared error between corresponding pixel values of the enhanced image and the input image. In a grayscale image, this value is equal to 255. PSNR is evaluated in decibels and is inversely proportional to mean square error (MSE). With PSNR, a larger value indicates better image quality. The basic Structural Similarity Index (SSIM) algorithm compares the input and the enhanced images pixel by pixel. The higher SSIM value, the better image quality.

Image enhancement plays a vital role in image preprocessing step of vehicle license plate recognition methods. There are various image enhancement operators or masks which vary from one another. The Riesz fractional differential operators and Regularized Extreme Learning Regressor are used extensively in image enhancement techniques because both have high SSIM values when compared to other techniques. Even though Riesz fractional operator has less PSNR value its high SSIM value enhances edge pixels such that structures of the characters would be preserved. Considering the benefits and limitations of the above mentioned techniques, it is notable that image enhancement techniques are more suitable than image super-resolution techniques for improving the low quality images.

\section{INFERENCE}

In this paper a comparison and analysis of different LPR systems in terms of their detection ratios is done and different problems faced by license plate detection methods are discussed. Super-resolution and enhancement techniques are discussed to overcome those problems. The future work will be on working with enhancement techniques rather than super-resolution techniques. The objective of this paper is to review about different LPD methods. Some LPR systems works under uncontrolled environment but some in restricted environment. License plate recognition system mainly consists of four steps such as vehicle image capture, license plate detection, character segmentation and character recognition. From the papers surveyed, it is realized that there are different methods and algorithms used for license plate detection, image enhancement and image super-resolutions based on various resolutions of images.

\section{REFERENCES}

[1] Yule Yuan, Member, IEEE, Wenbin Zou, Young Zhao, Xinan Wang, Xuefeng $\mathrm{Hu}$, and Nikos Komodakis, " A Robust and Efficient Approach to License Plate Detection", IEEE Transactions on image processing, Vol. 26, No. 3, pp. 1057-7149, March 2017.

[2] D. Zheng, Y. Zhao, and J. Wang, “ An efficient method of license plate location.”, Pattern Recognition Letter, Vol. 26, pp. 2431-2438, June 2005.

[3] Yong Zhao, Yule Yuan, Member, IEEE, Subin Bai, Kai Liu, Wei Fang, " Voting-based License Plate Location.”, International IEEE Conference on Intelligent Transportation Systems Washington, DC, USA, Oct 2011.

[4] Wengang Zhou, Houqiang Li, Yijuan Lu, Member, IEEE, and Qi Tian, Senior Member, IEEE, "Principal 
Visual Word Discovery for Automatic License Plate Detection.", IEEE Transactions on image processing, Vol. 21, No. 9, pp. 1057-7149, September 2012.

[5] Bo Li, Bin Tian, Ye Li, and Ding Wen, Senior Member, IEEE, "Component-Based-Based License Plate Detection Using Conditional Random Field Model.", IEEE Transactions on Intelligent Transportation Systems, Vol. 14, No. 4, pp. 1524-9050, Dec 2013.

[6] Runmin Wang, Nong Sang, Rui Huang, Yuehuan Wang, "License plate detection using gradient information and cascade detectors", Optik, Vol. 125, No. 1, pp. 186-190, 2014.

[7] Shan Du, Member, IEEE, Mahmoud Ibrahim, Mohamed Shehata, Senior Member, IEEE and Wael Badawy, Senior Member, IEEE, "Automatic License Plate Recognition(ANPR): A State-of-the-Art Review", IEEE Transactions on Circuits and systems for Video Technology, Vol. 23, No. 2, pp. 1051-8215, Feb 2013.

[8] Kai Zhang, Baoquan Wang, Wangmeng Zuo, Senior Member IEEE, Hongzhi Zhang, Member IEEE, Lei Zhang, Senior Member IEEE, "Joint Learning of Multiple Regressors for Single Image SuperResolution.", IEEE Transactions on Signal Processing Letters, Vol. 23, No. 1, pp. 1070-9908, Jan2016.
[9] Chao Dong, Chen Change Loy, Member, IEEE, Kaiming He, Member, IEEE, and Xiaoou Tang, Fellow, IEEE, "Image Super-Resolution Using Deep Convolutional Networks.”, IEEE Transaction on Pattern Analysis and Machine Intelligence, Vol. 38, No. 2, pp. 0162-8828, Feb 2016.

[10] Alexandre Nata Vicente, Helio Pedrini, "A LearningBased Single-Image Super-Resolution Method for Very Low Quality License Plate Images.”, IEEE International Conference on Systems, Oct 2016.

[11] K.S. Raghunandan, Palaiahnakote Shivakumara, Member IEEE, Hamid A. Jalab, Member IEEE, Rabha W. Ibrahim, Member IEEE, G. Hemantha Kumar, Umapada Pal, Senior Member IEEE and Tong Lu, Member IEEE, "Riesz Fractional Based Model for Enhancing License Plate Detection and Recognition", IEEE Personal use is permitted, pp. 1051-8215, 2016.

[12] Paheding Sidike, Evan Krieger, M. Zahangir Alom, Vijayan K. Asari and Tarek Taha, "A fast single-image super resolution via directional edge guided regularized extreme learning regression", Springer, Received: 15 March 2016/Revised: 7 September 2016/Accepted: 15 December 2016/ Published online: 11 Jan 2017.

[13] R. C. Gonzalez and R. E. Woods, Digital Image Processing, Second Edition, Pearson Education, 2002. 